\title{
A note on fractional integral operators on Herz spaces with variable exponent
}

Meng $\mathrm{Qu}^{*}$ and Jie Wang

\section{"Correspondence:}

qumeng@mail.ahnu.edu.cn

School of Mathematical and

Computer Sciences, Anhui Normal

University, Wuhu, 241003, China

\begin{abstract}
In this note, we prove that the fractional integral operators from Herz spaces with variable exponent $\dot{K}_{p(\cdot), q}^{\alpha}$ to Lipschitz-type spaces are bounded provided $p(\cdot)$ is locally log-Hölder continuous and log-Hölder continuous at infinity.
\end{abstract}

MSC: 42B20; 46E30

Keywords: Herz spaces; Lipschitz spaces; fractional integral; variable exponent

\section{Introduction}

Let $\mathbb{R}^{n}$ be the $n$-dimensional Euclid space. For $0<\beta<n$, the fractional integral operator $I_{\beta}$ is defined by

$$
I_{\beta} f(x)=\int_{\mathbb{R}^{n}} \frac{f(y)}{|x-y|^{n-\beta}} d y .
$$

The famous Hardy-Littlewood-Sobolev theorem tells us that $I_{\beta}$ is a bounded operator from the usual Lebesgue spaces $L^{p}$ to $L^{q}$ with $1 / q=1 / p-\beta / n$, where $1<p<n / \beta$. Also $I_{\beta}$ is bounded from $L^{\frac{n}{\beta}}$ into $B M O$. As for $p>n / \beta$, Gatto and Vagi [1] proved that $\tilde{I}_{\beta}$ is bounded from $L^{p}$ into Lipschitz spaces whose smoothness is controlled by $p$ and $\alpha$, where $\tilde{I}_{\beta}$ is defined as

$$
\tilde{I}_{\beta} f(x)=\int_{\mathbb{R}^{n}}\left(\frac{1}{|x-y|^{n-\beta}}-\frac{\chi_{\{|\cdot| \geq 1\}}(y)}{|y|^{n-\beta}}\right) f(y) d y
$$

Indeed Gatto and Vagi's result was proved in the setting of the spaces of homogeneous type. Also there are extensions like weighted function spaces theory, see [2]. Recently, Ramseyer et al. [3] extended Gatto and Vagi's result in the variable exponent function spaces case.

For the sake of convenience, we briefly recall some basic elements of the Lebesgue spaces with variable exponent, while more results can be found in $[4,5]$ and the references therein. Let $\Omega$ be a non-empty open set in $\mathbb{R}^{n}$ and $p(\cdot): \Omega \rightarrow[1, \infty)$ be a measurable function. The variable exponent Lebesgue space $L^{p(\cdot)}(\Omega)$ is defined by

$$
L^{p(\cdot)}(\Omega):=\left\{f \text { is measurable }: \int_{\Omega}\left|\frac{f(x)}{\lambda}\right|^{p(x)} d x<\infty \text { for some constant } \lambda>0\right\} .
$$

(c) 2015 Qu and Wang. This article is distributed under the terms of the Creative Commons Attribution 4.0 International License (http://creativecommons.org/licenses/by/4.0/), which permits unrestricted use, distribution, and reproduction in any medium, provided you give appropriate credit to the original author(s) and the source, provide a link to the Creative Commons license, and indicate if changes were made. 
It is easy to check that $L^{p(\cdot)}(\Omega)$ is a Banach space with the norm defined by

$$
\|f\|_{L^{p(\cdot)}(\Omega)}:=\inf \left\{\lambda>0: \int_{\Omega}\left|\frac{f(x)}{\lambda}\right|^{p(x)} d x \leq 1\right\} .
$$

We let

$$
p^{-}(\Omega):=\underset{x \in \Omega}{\operatorname{essinf}} p(x), \quad p^{+}(\Omega):=\underset{x \in \Omega}{\operatorname{ess} \sup } p(x),
$$

and we denote by $\mathcal{P}(\Omega)$ the set of measurable function $p(\cdot)$ on $\Omega$ with value in $[1, \infty)$ such that $1<p^{-}(\Omega) \leq p(\cdot) \leq p^{+}(\Omega)<\infty$. For the sake of simplicity, we write $L^{p(\cdot)}\left(\mathbb{R}^{n}\right)$ as $L^{p(\cdot)}$ and $\|f\|_{L^{p(\cdot)}\left(\mathbb{R}^{n}\right)}$ as $\|f\|_{p(\cdot)}$, respectively.

We say a function $p(\cdot): \mathbb{R}^{n} \longrightarrow \mathbb{R}$ is locally log-Hölder continuous, if there exists a constant $C$ such that

$$
|p(x)-p(y)| \leq \frac{C}{\log (e+1 /|x-y|)}
$$

for all $x, y \in \mathbb{R}^{n}$. If, for some $p_{\infty} \in \mathbb{R}$ and $C>0$, we have

$$
\left|p(x)-p_{\infty}\right| \leq \frac{C}{\log (e+|x|)}
$$

for all $x \in \mathbb{R}^{n}$, then we say $p(\cdot)$ is log-Hölder continuous at infinity.

The notation $\mathcal{P}^{\log }\left(\mathbb{R}^{n}\right)$ is used for all those exponents $p(\cdot) \in \mathcal{P}\left(\mathbb{R}^{n}\right)$ which are locally logHölder continuous and log-Hölder continuous at infinity with $p_{\infty}:=\lim _{|x| \rightarrow \infty} p(x)$. Moreover, we can easily show that $p(\cdot) \in \mathcal{P}^{\log }\left(\mathbb{R}^{n}\right)$ implies $p^{\prime}(\cdot) \in \mathcal{P}^{\log }\left(\mathbb{R}^{n}\right)$.

Ramseyer, Salinas and Viviani introduced the following function space, which can be viewed as the variable exponent counterpart of Lipschitz space defined by Peetre in [6].

Definition 1 ([3]) Let $0<\beta<n$ and $p(\cdot) \in \mathcal{P}\left(\mathbb{R}^{n}\right)$ and denote the Lebesgue measure of $B$ by $|B|$. We say that a locally integrable function $f$ belongs to $\mathcal{L}_{\beta, p(\cdot)}\left(\mathbb{R}^{n}\right)$ if there exists a constant $C$ such that

$$
\frac{1}{|B|^{\frac{\beta}{n}}\left\|\chi_{Q}\right\|_{p^{\prime}(\cdot)}} \int_{B}\left|f-m_{B} f\right| d x \leq C,
$$

for every ball $B=B(x, R) \subset \mathbb{R}^{n}$, with $m_{B} f=\frac{1}{|B|} \int_{B} f$. The least constant $C$ in (1.1) will be denoted by $\|f\|_{L i p_{\beta, p(\cdot)}}$.

Ramseyer, Salinas and Viviani proved the following theorem.

Theorem 1.1 ([3]) Given $0<\beta<n$ and $p(\cdot) \in \mathcal{P}\left(\mathbb{R}^{n}\right)$. Then the following two statements are equivalent.

(1) $\tilde{I}_{\beta}$ is bounded from $L^{p(\cdot)}\left(\mathbb{R}^{n}\right)$ into $\mathcal{L}_{\beta, p(\cdot)}\left(\mathbb{R}^{n}\right)$.

(2) $p(\cdot) \in P_{\beta}$, i.e., there exists a positive constant $C$ such that for any ball $B$,

$$
\left\|\frac{\chi_{\mathbb{R}^{n} \backslash B}}{\left|x_{B}-\cdot\right|^{n-\beta+1}}\right\|_{p^{\prime}(\cdot)} \leq C|B|^{\frac{\beta}{n}-\frac{1}{n}-1}\left\|\chi_{B}\right\|_{p^{\prime}(\cdot)}
$$

hold for every ball $B$, where $x_{B}$ denotes its center. 
Corollary 2.16 in [3] says that if $p(\cdot) \in \mathcal{P}^{\log }\left(\mathbb{R}^{n}\right)$ with $0<\beta-1<\frac{n}{p^{+}}$, then $p(\cdot)$ satisfies (1.2). With the help of Theorem 1.1, $\tilde{I}_{\beta}$ is bounded from $L^{p(\cdot)}\left(\mathbb{R}^{n}\right)$ to $\mathcal{L}_{\beta, p(\cdot)}\left(\mathbb{R}^{n}\right)$. It is natural to ask what the target space is when $L^{p(\cdot)}\left(\mathbb{R}^{n}\right)$ is replaced by other more general spaces. The main result of this note is that the target space of mapping $\tilde{I}_{\beta}$ is just the variant Lipschitz space when $L^{p(\cdot)}\left(\mathbb{R}^{n}\right)$ is replaced by the so-called variable exponent Herz space.

\section{Herz spaces and main results}

Variable exponent Herz spaces were considered by many authors in recent years. Especially Herz spaces with two variable exponents and even with three variable exponents were produced by Almeida and Drihem [7] and Samko [8], respectively. For brevity, we only consider the Herz space with one variable exponent case, which was introduced by Izuki in [9]. Let $B(x, r)=\left\{y \in \mathbb{R}^{n}:|x-y|<r\right\}, B_{k}=\left\{x \in \mathbb{R}^{n}:|x|<2^{k}\right\}, A_{k}=B_{k} \backslash B_{k-1}$, and $\chi_{A_{k}}=\chi_{k}$ be the characteristic function of the set $A_{k}$ for $k \in \mathbb{Z}$.

Definition 2 ([9]) Let $\alpha \in \mathbb{R}, 0<q \leq \infty$ and $p(\cdot) \in \mathcal{P}\left(\mathbb{R}^{n}\right)$. The homogeneous Herz space $\dot{K}_{p(\cdot), q}^{\alpha}\left(\mathbb{R}^{n}\right)$ is defined as the set of all $f \in L_{\text {loc }}^{p(\cdot)}\left(\mathbb{R}^{n} \backslash\{0\}\right)$ such that

$$
\|f\|_{\dot{K}_{p(\cdot), q}^{\alpha}\left(\mathbb{R}^{n}\right)}:=\left(\sum_{k \in \mathbb{Z}} 2^{k \alpha q}\left\|f \chi_{k}\right\|_{p(\cdot)}^{q}\right)^{1 / q}<\infty .
$$

It is obvious that if $p(\cdot)$ is a constant, then $\dot{K}_{p(\cdot), q}^{\alpha}\left(\mathbb{R}^{n}\right)=\dot{K}_{p, q}^{\alpha}\left(\mathbb{R}^{n}\right)$ are classical Herz spaces. We can refer to [10] for more properties of the classical one.

Our main result is to establish a result of mapping property of $\tilde{I}_{\beta}$ on $\dot{K}_{p(\cdot), q}^{\alpha}\left(\mathbb{R}^{n}\right)$. For this purpose we need to define a variant of the Lipschitz space.

Definition 3 Given $-\infty<\lambda<+\infty, 0<\beta<n$, and $p(\cdot) \in \mathcal{P}\left(\mathbb{R}^{n}\right)$. We say that a locally integrable function $f$ belongs to $\mathcal{L}_{\beta, p(.)}^{\lambda}$ if there exists a constant $C$ such that

$$
\frac{1}{(|x|+R)^{\lambda}} \frac{1}{|B|^{\frac{\beta}{n}}\left\|\chi_{B}\right\|_{p^{\prime}(\cdot)}} \int_{B}\left|f-m_{B} f\right| d x \leq C,
$$

for every ball $B=B(x, R) \subset \mathbb{R}^{n}$, with $m_{B} f=\frac{1}{|B|} \int_{B} f$. The least constant $C$ in (2.1) will be denoted by $\|f\|_{\mathcal{L}_{\beta, p(\cdot)}^{\lambda}}$.

Remark 2.1 It is easy to see that in Definition 3 the average $m_{B} f$ can be replaced by a constant in the following sense:

$$
\frac{1}{2}\|f\|_{L i p_{\beta, p(\cdot)}^{\lambda}} \leq \sup _{B \in \mathbb{R}^{n}, R>0} \inf _{c} \frac{1}{(|x|+R)^{\lambda}} \frac{1}{|B|^{\frac{\beta}{n}}\left\|\chi_{B}\right\|_{p^{\prime}(\cdot)}} \int_{B}|f-c| d x \leq\|f\|_{L i p_{\beta, p(\cdot)}^{\lambda}} .
$$

Also by Definition 3, we obtain $\mathcal{L}_{\beta_{1}, p(\cdot)}^{\lambda_{1}} \subset \mathcal{L}_{\beta, p(\cdot)}^{\lambda}$, where $\lambda-\lambda_{1}=\beta_{1}-\beta \geq 0$. Especially, $\mathcal{L}_{\beta, p(\cdot)}^{\lambda} \subset \mathcal{L}_{\beta+\lambda, p(\cdot)}$ for $\lambda<0$ and $\mathcal{L}_{\beta+\lambda, p(\cdot)} \subset \mathcal{L}_{\beta, p(\cdot)}^{\lambda}$ for $\lambda>0$.

Now we are in a position to state our results.

Theorem 2.1 Suppose that $0<q<\infty$ and $p(\cdot) \in \mathcal{P}^{\log }\left(\mathbb{R}^{n}\right)$. If $\beta-\frac{n}{p^{+}}-1<\alpha<n-\frac{n}{p^{-}}, 1<$ $\beta<\frac{n}{p^{+}}+1$, then the operator $\tilde{I}_{\beta}$ is bounded from $\dot{K}_{p(\cdot), q}^{\alpha}\left(\mathbb{R}^{n}\right)$ to $\mathcal{L}_{\beta, p(\cdot)}^{-\alpha}\left(\mathbb{R}^{n}\right)$. 
Theorem 2.2 Suppose that $0<q<\infty$ and $p(x) \in \mathcal{P}^{\log }\left(\mathbb{R}^{n}\right)$. If $\varepsilon>0$, then $\tilde{I}_{\beta}$ is not bounded from $\dot{K}_{p(\cdot), q}^{\alpha}\left(\mathbb{R}^{1}\right)$ to $\mathcal{L}_{\beta+\varepsilon, p(\cdot)}^{-\alpha-\varepsilon}\left(\mathbb{R}^{1}\right)$.

Remark 2.2 According to Remark 2.1, $\mathcal{L}_{\beta+\varepsilon, p(\cdot)}^{-\alpha-\varepsilon}\left(\mathbb{R}^{1}\right) \subset \mathcal{L}_{\beta, p(\cdot)}^{-\alpha}\left(\mathbb{R}^{1}\right)$ when $\varepsilon>0$. This shows that Theorem 2.1 is optimal.

We give some lemmas in Section 3 and then prove the above theorems in Section 4. $C$ always means a positive constant independent of the main parameters and it may change from one occurrence to another. $f \sim g$ means $C^{-1} g \leq f \leq C g$.

\section{Technique lemmas}

Lemma 3.1 ([11]) Let $\Omega \subset \mathbb{R}^{n}$. If $p(\cdot) \in \mathcal{P}(\Omega)$, then for all $f \in L^{p(\cdot)}(\Omega)$ and all $g \in L^{p^{\prime} \cdot(\cdot)}(\Omega)$ we have

$$
\int_{\Omega}|f(x) g(x)| d x \leq r_{p}\|f\|_{L^{p(\cdot)}(\Omega)}\|g\|_{L^{p^{(\cdot)}(\Omega)}},
$$

where $r_{p}:=1+\frac{1}{\left.p^{-(} \Omega\right)}-\frac{1}{p^{+}(\Omega)}$.

Given a function $f \in L_{\mathrm{loc}}^{1}\left(\mathbb{R}^{n}\right)$, the Hardy-Littlewood maximal operator $M$ is defined by

$$
M f(x):=\sup _{r>0} r^{-n} \int_{B(x, r)}|f(y)| d y, \quad x \in \mathbb{R}^{n},
$$

and we say $\mathcal{B}\left(\mathbb{R}^{n}\right)$ is the set of $p(\cdot) \in \mathcal{P}\left(\mathbb{R}^{n}\right)$ satisfying the condition that $M$ is bounded on $L^{p(\cdot)}\left(\mathbb{R}^{n}\right)$.

Lemma 3.2 ([12]) $p(\cdot) \in \mathcal{P}^{\log }\left(\mathbb{R}^{n}\right)$ implies $p(\cdot) \in \mathcal{B}\left(\mathbb{R}^{n}\right)$.

Lemma 3.3 ([13]) Let $p(\cdot) \in \mathcal{B}\left(\mathbb{R}^{n}\right)$, then there exists a positive constant $C$ such that

$$
C^{-1}|B| \leq\left\|\chi_{B}\right\|_{p(\cdot)}\left\|\chi_{B}\right\|_{p^{\prime}(\cdot)} \leq C|B|
$$

hold for every ball $B$.

Remark 3.1 According to Lemma 3.2, the conclusion of Lemma 3.3 is correct when the condition $p(\cdot) \in \mathcal{B}\left(\mathbb{R}^{n}\right)$ is replaced by $p(\cdot) \in \mathcal{P}^{\log }\left(\mathbb{R}^{n}\right)$.

Lemma 3.4 (Corollary 4.5.9 in [5]) Let $p(\cdot) \in \mathcal{P}^{\log }\left(\mathbb{R}^{n}\right)$, then for every ball $B \subset \mathbb{R}^{n}$,

$$
\left\|\chi_{B}\right\|_{p(\cdot)} \sim|B|^{\frac{1}{p(x)}}, \quad \text { if }|B| \leq 2^{n}, x \in B
$$

and

$$
\left\|\chi_{B}\right\|_{p(\cdot)} \sim|B|^{\frac{1}{p_{\infty}}}, \quad \text { if }|B| \geq 1
$$

Lemma 3.5 ([3]) Let $p(\cdot) \in \mathcal{P}\left(\mathbb{R}^{n}\right)$ and $0<\beta-1<\frac{n}{p^{+}}$.

(1) If $p(\cdot) \in \mathcal{P}^{\log }\left(\mathbb{R}^{n}\right)$ and $0<\beta-1<\frac{n}{p^{+}}$, then $p(\cdot) \in P_{\beta}$. 
(2) If $p(\cdot) \in P_{\beta}$, then there exists a positive constant $C$ such that

$$
\left\|\chi_{2 B}\right\|_{p(\cdot)} \leq C\left\|\chi_{B}\right\|_{p(\cdot)}
$$

for every ball $B$, where $2 B$ is the ball having the same center as $B$ but whose diameter is two times as large.

We point out that the two results collected in Lemma 3.5 are from [3]. The result (1) is Corollary 2.16 and (2) is Lemma 2.9 therein, respectively.

Lemma 3.6 Let $p(\cdot) \in \mathcal{P}^{\log }\left(\mathbb{R}^{n}\right)$, then there exists a constant $C>0$ such that for all balls $B$ and all measurable subsets $S=B\left(x_{0}, r_{0}\right) \subset B=B\left(x_{1}, r_{1}\right)$,

$$
\frac{\left\|\chi_{S}\right\|_{p(\cdot)}}{\left\|\chi_{B}\right\|_{p(\cdot)}} \leq C\left(\frac{|S|}{|B|}\right)^{\frac{1}{p^{+}}} .
$$

Proof We proved the lemma in the following three cases: (1) $|S|<|B|<1$; (2) $|S|<1<$ $|B|$; (3) $1 \leq|S|<|B|$. Cases (2) and (3) are easy, we omit the details. Now for case (1). By Lemma 3.4,

$$
\frac{\left\|\chi_{S}\right\|_{p(\cdot)}}{\left\|\chi_{B}\right\|_{p(\cdot)}} \sim \frac{|S|^{\frac{1}{p\left(x_{S}\right)}}}{|B|^{\frac{1}{p\left(x_{S}\right)}}}|B|^{\frac{1}{p\left(x_{S}\right)}}-\frac{1}{p\left(x_{B}\right)} \leq C\left(\frac{|S|}{|B|}\right)^{\frac{1}{p^{+}}} .
$$

Indeed in the last inequality in the above equation, since $\left|x_{B}-x_{S}\right| \leq 2 r_{1}$, we make use of the local-Hölder continuity of $p(x)$, so

$$
\begin{aligned}
\left|\frac{1}{p\left(x_{S}\right)}-\frac{1}{p\left(x_{B}\right)}\right| \log \frac{1}{r_{1}} & \leq \frac{\log \frac{1}{r_{1}}}{\log \left(e+\frac{1}{\left|x_{S}-x_{B}\right|}\right)} \\
& \leq \frac{\log \frac{1}{r_{1}}}{\log \left(e+\frac{1}{2 r_{1}}\right)} \leq C .
\end{aligned}
$$

The lemma is proved.

\section{Proofs of theorems}

Proof of Theorem 2.1 Fix a ball $Q=B\left(x_{0}, R\right)$. To prove Theorem 2.1, we need to estimate

$$
\frac{\left(\left|x_{0}\right|+R\right)^{\alpha}}{|Q|^{\frac{\beta}{n}}\left\|\chi_{Q}\right\|_{p^{\prime}(\cdot)}} \int_{Q}\left|\tilde{I}_{\beta} f(x)-c\right| d x .
$$

Let $k$ be the least integer such that $Q \subset B\left(0,2^{k}\right)$, hence $\left|x_{0}\right|+R \sim 2^{k}$. We consider three cases:

(1) $Q \cap B\left(0,2^{k-2}\right) \neq \emptyset$,

(2) $Q \cap B\left(0,2^{k-2}\right)=\emptyset$ and $R \geq 2^{k-4}$,

(3) $Q \cap B\left(0,2^{k-2}\right)=\emptyset$ and $R<2^{k-4}$. 
Case (1) or (2). Note that $|Q| \geq C 2^{k n}$ in both cases. We write

$$
f(x)=f \chi_{B\left(0,2^{k+1}\right)}(x)+f \chi_{\mathbb{R}^{n} \backslash B\left(0,2^{k+1}\right)}(x)=: f_{1}(x)+f_{2}(x) .
$$

First we estimate $\tilde{I}_{\beta} f_{1}$.

Let $c_{1}=-\int_{|y| \geq 1} \frac{f_{1}(y)}{|y|^{n-\beta}} d y$, then $\tilde{I}_{\beta} f_{1}-c_{1}=I_{\beta} f_{1}$. For any $x \in Q,\left|I_{\beta} f_{1}(x)\right| \leq \int_{B\left(0,2^{k+1}\right)} \frac{|f(y)|}{|x-y|^{n-\beta}} d y$. Then by Fubini's theorem, we have

$$
\begin{aligned}
\int_{Q}\left|I_{\beta} f_{1}(x)\right| d x & \leq \int_{B\left(0,2^{k+1}\right)}|f(y)| \int_{Q} \frac{1}{|x-y|^{n-\beta}} d x d y \\
& \leq C|Q|^{\frac{\beta}{n}} \int_{B\left(0,2^{k+1}\right)}|f(y)| d y \\
& =C|Q|^{\frac{\beta}{n}} \sum_{j=-\infty}^{k+1} \int_{A_{j}}|f(y)| d y .
\end{aligned}
$$

Using Lemma 3.1 and Lemma 3.3, we derive the estimate

$$
\begin{aligned}
\frac{\left(\left|x_{0}\right|+R\right)^{\alpha}}{|Q|^{\frac{\beta}{n}}\left\|\chi_{Q}\right\|_{p^{\prime}(\cdot)}} \int_{Q}\left|I_{\beta} f_{1}(x)\right| d x & \leq C \sum_{j=-\infty}^{k+1} \frac{\left(\left|x_{0}\right|+R\right)^{\alpha}}{\left\|\chi_{Q}\right\|_{p^{\prime}(\cdot)}} \int_{A_{j}}|f(y)| d y \\
& \leq C \sum_{j=-\infty}^{k+1} 2^{k \alpha} \frac{\left\|\chi_{Q}\right\|_{p(\cdot)}}{|Q|}\left\|f \chi_{j}\right\|_{p(\cdot)}\left\|\chi_{j}\right\|_{p^{\prime}(\cdot)} \\
& \leq C \sum_{j=-\infty}^{k+1} 2^{k \alpha} 2^{-k n}\left\|f \chi_{j}\right\|_{p(\cdot)}\left\|\chi_{B_{j}}\right\|_{p^{\prime}(\cdot)}\left\|\chi_{B_{k}}\right\|_{p(\cdot) \cdot}
\end{aligned}
$$

Now we can distinguish three cases as follows, by Lemma 3.4:

(1) If $0 \leq j-1 \leq k$, we have

$$
\begin{aligned}
\left\|\chi_{B_{j}}\right\|_{p^{\prime}(\cdot)}\left\|\chi_{B_{k}}\right\|_{p(\cdot)} & \sim\left|B_{j}\right|^{\frac{1}{p_{\infty}^{\prime}}}\left|B_{k}\right|^{\frac{1}{p_{\infty}}} \sim\left(2^{j n}\right)^{\frac{1}{p_{\infty}^{\prime}}}\left(2^{k n}\right)^{\frac{1}{p_{\infty}}} \\
& \sim 2^{j n} 2^{(k-j) \frac{n}{p_{\infty}}} \leq C 2^{j n} 2^{(k-j) \frac{n}{p^{-}}} .
\end{aligned}
$$

(2) If $j-1<0 \leq k$, we obtain

$$
\begin{aligned}
\left\|\chi_{B_{j}}\right\|_{p^{\prime}(\cdot)}\left\|\chi_{B_{k}}\right\|_{p(\cdot)} & \sim\left|B_{j}\right|^{\frac{1}{p^{\prime}\left(x_{j}\right)}}\left|B_{k}\right|^{\frac{1}{p \infty}} \sim\left(2^{j n}\right)^{\frac{1}{p^{\prime}\left(x_{j}\right)}}\left(2^{k n}\right)^{\frac{1}{p \infty}} \\
& \sim 2^{j n}\left(2^{-j n}\right)^{\frac{1}{p\left(x_{j}\right)}}\left(2^{k n}\right)^{\frac{1}{p \infty}} \leq C 2^{j n} 2^{(k-j) \frac{n}{p^{-}}} .
\end{aligned}
$$

(3) If $j-1 \leq k<0$, we get

$$
\begin{aligned}
\left\|\chi_{B_{j}}\right\|_{p^{\prime}(\cdot)}\left\|\chi_{B_{k}}\right\|_{p(\cdot)} & \sim\left|B_{j}\right|^{\frac{1}{p^{\prime}\left(x_{j}\right)}}\left|B_{k}\right|^{\frac{1}{p\left(x_{k}\right)}} \sim\left(2^{j n}\right)^{\frac{1}{p^{\prime}\left(x_{j}\right)}}\left(2^{k n}\right)^{\frac{1}{p\left(x_{k}\right)}} \\
& \sim 2^{j n} 2^{(k-j) \frac{n}{p\left(x_{j}\right)}}\left(2^{k n}\right)^{\frac{1}{p\left(x_{k}\right)}-\frac{1}{p\left(x_{j}\right)}} \leq C 2^{j n} 2^{(k-j) \frac{n}{p^{-}}} .
\end{aligned}
$$


Here in the last inequality we using the following facts: If $k \geq 0,\left|x_{k}\right|<2^{k}$, and $\left|x_{j}\right|<2^{j} \leq 2^{k}$, then the local-Hölder continuity of $p(x)$ at the origin yields

$$
\begin{aligned}
\left|\frac{1}{p\left(x_{k}\right)}-\frac{1}{p\left(x_{j}\right)}\right| \log \frac{1}{2^{k}} & \leq\left|\frac{1}{p\left(x_{k}\right)}-\frac{1}{p(0)}\right| \log \frac{1}{2^{k}}+\left|\frac{1}{p\left(x_{j}\right)}-\frac{1}{p(0)}\right| \log \frac{1}{2^{k}} \\
& \leq C \frac{\log \frac{1}{2^{k}}}{\log \left(e+\frac{1}{2^{k}}\right)} \leq C
\end{aligned}
$$

with $C>0$ independent of $k, j, x_{k}, x_{j}$.

Therefore,

$$
\frac{\left(\left|x_{0}\right|+R\right)^{\alpha}}{|Q|^{\frac{\beta}{n}}\left\|\chi_{Q}\right\|_{p^{\prime}(\cdot)}} \int_{Q}\left|I_{\beta} f_{1}(x)\right| d x \leq C \sum_{j=-\infty}^{k+1} 2^{(k-j)\left(\alpha-n+\frac{n}{p^{-}}\right)} 2^{j \alpha}\left\|f \chi_{j}\right\|_{p(\cdot)}
$$

Since

$$
2^{j \alpha}\left\|f \chi_{j}\right\|_{L^{p(\cdot)}\left(\mathbb{R}^{n}\right)}=\left(2^{j q \alpha}\left\|f \chi_{j}\right\|_{L^{p \cdot(\cdot)}\left(\mathbb{R}^{n}\right)}^{q}\right)^{1 / q} \leq\left(\sum_{i=-\infty}^{\infty} 2^{i q \alpha}\left\|f \chi_{i}\right\|_{L^{p}(\cdot)}^{q}\left(\mathbb{R}^{n}\right)\right)^{1 / q} \leq\|f\|_{\dot{K}_{p(\cdot), q^{\alpha}}^{\alpha}\left(\mathbb{R}^{n}\right)} .
$$

Thus by the condition $\alpha-n+\frac{n}{p^{-}}<0$, it follows that

$$
\frac{\left(\left|x_{0}\right|+R\right)^{\alpha}}{|Q|^{\frac{\beta}{n}}\left\|\chi_{Q}\right\|_{p^{\prime}(\cdot)}} \int_{Q}\left|I_{\beta} f_{1}(x)\right| d x \leq C\|f\|_{K_{p(\cdot), q}^{\alpha}\left(\mathbb{R}^{n}\right)} .
$$

Next we estimate $\tilde{I}_{\beta} f_{2}$. Let $c_{2}=\tilde{I}_{\beta} f_{2}\left(x_{0}\right)$. For any $x \in Q, y \in A_{j}$ and $j \geq k+2$, we have $\left|x_{0}-y\right| \geq|y|-\left|x_{0}\right|>2^{j-1}-2^{k} \geq 2^{j-2}$. Then

$$
\begin{aligned}
\left|\tilde{I}_{\beta} f_{2}(x)-c_{2}\right| & \leq \int_{\mathbb{R}^{n}}\left|\frac{1}{|x-y|^{n-\beta}}-\frac{1}{\left|x_{0}-y\right|^{n-\beta}}\right|\left|f_{2}(y)\right| d y \\
& \leq C R \int_{\mathbb{R}^{n} \backslash B\left(0,2^{k+1}\right)} \frac{|f(y)|}{\left|x_{0}-y\right|^{n-\beta+1}} d y \\
& \leq C R \sum_{j=k+2}^{\infty} \int_{A_{j}} \frac{|f(y)|}{\left|x_{0}-y\right|^{n-\beta+1}} d y \\
& \leq C R \sum_{j=k+2}^{\infty} 2^{-j(n-\beta+1)} \int_{A_{j}}|f(y)| d y .
\end{aligned}
$$

By Lemma 3.1 and Lemma 3.3, we obtain

$$
\begin{aligned}
\frac{\left(\left|x_{0}\right|+R\right)^{\alpha}}{|Q|^{\frac{\beta}{n}}\left\|\chi_{Q}\right\|_{p^{\prime}(\cdot)}} \int_{Q}\left|\tilde{I}_{\beta} f_{2}(x)-c_{2}\right| d x & \leq C R \sum_{j=k+2}^{\infty} \frac{2^{k \alpha} 2^{-j(n-\beta+1)}}{|Q|^{\frac{\beta}{n}}\left\|\chi_{Q}\right\|_{p^{\prime}(\cdot)}}|Q| \int_{A_{j}}|f(y)| d y \\
& \leq C R \sum_{j=k+2}^{\infty} \frac{2^{k \alpha} 2^{-j(n-\beta+1)}\left\|\chi_{Q}\right\|_{p(\cdot)}}{|Q|^{\frac{\beta}{n}}}\left\|f \chi_{j}\right\|_{\left.p^{(\cdot)}\right)}\left\|\chi_{j}\right\|_{p^{\prime}(\cdot)} \\
& \leq C R \sum_{j=k+2}^{\infty} \frac{2^{k \alpha} 2^{-j(n-\beta+1)}}{2^{k \beta}}\left\|f \chi_{j}\right\|_{p(\cdot)}\left\|\chi_{B_{j}}\right\|_{p^{\prime}(\cdot)}\left\|\chi_{B_{k}}\right\|_{p(\cdot)} .
\end{aligned}
$$


Applying the arguments used in the corresponding step of the estimate of $\tilde{I}_{\beta} f_{1}$, we arrive at the inequality

$$
\left\|\chi_{B_{j}}\right\|_{p^{\prime}(\cdot)}\left\|\chi_{B_{k}}\right\|_{p(\cdot)} \leq C 2^{j n} 2^{(k-j) \frac{n}{p^{+}}} .
$$

Since $\alpha-\beta+\frac{n}{p^{+}}+1>0$,

$$
\begin{aligned}
\frac{\left(\left|x_{0}\right|+R\right)^{\alpha}}{|Q|^{\frac{\beta}{n}}\left\|\chi_{Q}\right\|_{p^{\prime}(\cdot)}} \int_{Q}\left|\tilde{I}_{\beta} f_{2}(x)-c_{2}\right| d x & \leq C \sum_{j=k+2}^{\infty} 2^{(k-j)\left(\alpha-\beta+\frac{n}{\left.p^{+}+1\right)}\right.} 2^{j \alpha}\left\|f \chi_{j}\right\|_{p(\cdot)} \\
& \leq C\|f\|_{\dot{K}_{p(\cdot), q}^{\alpha}\left(\mathbb{R}^{n}\right)} .
\end{aligned}
$$

Combining (4.1)-(4.5), cases (1) and (2) are proved.

Case (3). We write

$$
\begin{aligned}
f(x) & =f \chi_{B\left(x_{0}, 2 R\right)}(x)+f \chi_{B_{k+1} \backslash\left(B_{k-3} \cup B\left(x_{0}, 2 R\right)\right)}(x)+f \chi_{B_{k-3}}(x)+f \chi_{\mathbb{R}^{n} \backslash B_{k+1}}(x) \\
& =: f_{1}(x)+f_{2}(x)+f_{3}(x)+f_{4}(x) .
\end{aligned}
$$

First we estimate $\tilde{I}_{\beta} f_{1}$. Let $c_{1}=-\int_{|y| \geq 1} \frac{f_{1}(y)}{|y|^{n-\beta}} d y$, then $\tilde{I}_{\beta} f_{1}-c_{1}=I_{\beta} f_{1}$. For any $x \in Q,\left|I_{\beta} f_{1}(x)\right| \leq$ $\int_{B\left(x_{0}, 2 R\right)} \frac{|f(y)|}{\left.|x-y|\right|^{n-\beta}} d y$. Then by Fubini's theorem and Lemma 3.1, we obtain

$$
\begin{aligned}
\int_{Q}\left|I_{\beta} f_{1}(x)\right| d x & \leq \int_{B\left(x_{0}, 2 R\right)}|f(y)| \int_{Q} \frac{1}{|x-y|^{n-\beta}} d x d y \\
& \leq C|Q|^{\frac{\beta}{n}} \int_{B\left(x_{0}, 2 R\right)}|f(y)| d y \\
& \leq C|Q|^{\frac{\beta}{n}}\left\|f \chi_{B\left(x_{0}, 2 R\right)}\right\|_{p(\cdot)}\left\|\chi_{B\left(x_{0}, 2 R\right)}\right\|_{p^{\prime}(\cdot) .}
\end{aligned}
$$

Note that $B\left(x_{0}, 2 R\right) \subset \bigcup_{j=k-2}^{k+1} A_{j}$, so

$$
\begin{aligned}
\frac{\left(\left|x_{0}\right|+R\right)^{\alpha}}{|Q|^{\frac{\beta}{n}}\left\|\chi_{Q}\right\|_{p^{\prime}(\cdot)}} \int_{Q}\left|\tilde{I}_{\beta} f_{1}(x)-c_{1}\right| d x & \leq C 2^{k \alpha}\left\|f \chi_{B\left(x_{0}, 2 R\right)}\right\|_{p(\cdot)} \\
& \leq C \sum_{j=k-2}^{k+1} 2^{j \alpha}\left\|f \chi_{j}\right\|_{p(\cdot)} \\
& \leq C\|f\|_{\dot{K}_{p(\cdot), q}^{\alpha}\left(\mathbb{R}^{n}\right)} .
\end{aligned}
$$

Next we estimate $\tilde{I}_{\beta} f_{2}$. Let $c_{2}=\tilde{I}_{\beta} f_{2}\left(x_{0}\right)$. By Lemma 3.1 , and then by the condition $1<\beta<$ $\frac{n}{p^{+}}+1$ with Lemma 3.5 ,

$$
\begin{aligned}
\left|\tilde{I}_{\beta} f_{2}(x)-c_{2}\right| & \leq C R \int_{\left|x_{0}-y\right|>2 R} \frac{\left|f_{2}(y)\right|}{\left|x_{0}-y\right|^{n-\beta+1}} d y \\
& \leq C R\left\|f \chi_{B_{k+1} \backslash B_{k-3}}\right\|_{p(\cdot)}\left\|\frac{\chi_{\mathbb{R}^{n} \backslash B\left(x_{0}, 2 R\right)}}{\left|x_{0}-y\right|^{n-\beta+1}}\right\|_{p^{\prime}(\cdot)} \\
& \leq C\left|B\left(x_{0}, 2 R\right)\right|^{\frac{\beta}{n}-1}\left\|f \chi_{B_{k+1} \backslash B_{k-3}}\right\|_{p(\cdot)}\left\|\chi_{B\left(x_{0}, 2 R\right)}\right\|_{p^{\prime}(\cdot)} \\
& \leq C|Q|^{\frac{\beta}{n}-1}\left\|f \chi_{B_{k+1} \backslash B_{k-3}}\right\|_{p(\cdot)}\left\|\chi_{Q}\right\|_{p^{\prime}(\cdot)} .
\end{aligned}
$$


Hence

$$
\begin{aligned}
\frac{\left(\left|x_{0}\right|+R\right)^{\alpha}}{|Q|^{\frac{\beta}{n}}\left\|\chi_{Q}\right\|_{p^{\prime}(\cdot)}} \int_{Q}\left|\tilde{I}_{\beta} f_{2}(x)-c_{2}\right| d x & \leq C 2^{k \alpha}\left\|f \chi_{B_{k+1} \backslash B_{k-3}}\right\|_{p(\cdot)} \\
& \leq C\|f\|_{\dot{K}_{p(\cdot), q}^{\alpha}}\left(\mathbb{R}^{n}\right)
\end{aligned}
$$

Now we estimate $\tilde{I}_{\beta} f_{4}$. Let $c_{4}=\tilde{I}_{\beta} f_{4}\left(x_{0}\right)$. For any $x \in Q, y \in B\left(0,2^{k-3}\right)$, we have $\left|x_{0}-y\right| \geq$ $\left|x_{0}\right|-|y|>2^{k-2}-2^{k-3}=2^{k-3}$. Then

$$
\begin{aligned}
\left|\tilde{I}_{\beta} f_{4}(x)-c_{4}\right| & \leq \int_{\mathbb{R}^{n}}\left|\frac{1}{|x-y|^{n-\beta}}-\frac{1}{\left|x_{0}-y\right|^{n-\beta}}\right|\left|f_{4}(y)\right| d y \\
& \leq C R \int_{B\left(0,2^{k-3}\right)} \frac{|f(y)|}{\left|x_{0}-y\right|^{n-\beta+1}} d y \\
& \leq C R 2^{-k(n-\beta+1)} \int_{B\left(0,2^{k-3}\right)}|f(y)| d y \\
& =C R 2^{-k(n-\beta+1)} \sum_{j=-\infty}^{k-3} \int_{A_{j}}|f(y)| d y .
\end{aligned}
$$

Now Lemma 3.1 yields

$$
\left|\tilde{I}_{\beta} f_{4}(x)-c_{4}\right| \leq C R 2^{-k(n-\beta+1)} \sum_{j=-\infty}^{k-3}\left\|f \chi_{j}\right\|_{p(\cdot)}\left\|\chi_{j}\right\|_{p^{\prime}(\cdot)}
$$

Lemma 3.6 gives

$$
\frac{\left\|\chi_{Q}\right\|_{p(\cdot)}}{\left\|\chi_{B_{k}}\right\|_{p(\cdot)}} \leq C\left(\frac{|Q|}{\left|B_{k}\right|}\right)^{\frac{1}{p^{+}}}
$$

Since $\alpha-n+\frac{n}{p^{-}}<0$, we have

$$
\begin{aligned}
& \frac{\left(\left|x_{0}\right|+R\right)^{\alpha}}{|Q|^{\frac{\beta}{n}}\left\|\chi_{Q}\right\|_{p^{\prime}(\cdot)}} \int_{Q}\left|\tilde{I}_{\beta} f_{4}(x)-c_{4}\right| d x \\
& \leq C \frac{2^{k \alpha}}{|Q|^{\frac{\beta-1}{n}}} 2^{-k(n-\beta+1)} \sum_{j=-\infty}^{k-3}\left\|f \chi_{j}\right\|_{p(\cdot)}\left\|\chi_{j}\right\|_{p^{\prime}(\cdot)}\left\|\chi_{Q}\right\|_{p(\cdot)} \\
& \leq C \frac{2^{k \alpha}}{\left|B_{k}\right|^{\frac{\beta-1}{n}}} 2^{-k(n-\beta+1)}\left(\frac{|Q|}{\left|B_{k}\right|}\right)^{\frac{1}{p^{+}-\frac{\beta-1}{n}}} \sum_{j=-\infty}^{k-3}\left\|f \chi_{j}\right\|_{p(\cdot)}\left\|\chi_{B_{j}}\right\|_{p^{\prime}(\cdot)}\left\|\chi_{B_{k}}\right\|_{p(\cdot)} \\
& \leq C \sum_{j=-\infty}^{k-3} 2^{(k-j)\left(\alpha-n+\frac{n}{\left.p^{-}\right)}\right.} 2^{j \alpha}\left\|f \chi_{j}\right\|_{p(\cdot)} \\
& \leq C\|f\|_{K_{p(\cdot), q}^{\alpha}}\left(\mathbb{R}^{n}\right) .
\end{aligned}
$$


Finally we estimate $\tilde{I}_{\beta} f_{3}$. Let $c_{3}=\tilde{I}_{\beta} f_{3}\left(x_{0}\right)$. For any $x \in Q, y \in \mathbb{R}^{n} \backslash B\left(0,2^{k+1}\right)$, and $j \geq k+2$, we have $\left|x_{0}-y\right| \geq|y|-\left|x_{0}\right|>2^{k+1}-2^{k}=2^{k}$. Then we write

$$
\begin{aligned}
\left|\tilde{I}_{\beta} f_{3}(x)-c_{3}\right| & \leq C R \int_{\mathbb{R}^{n} \backslash B\left(0,2^{k+1}\right)} \frac{|f(y)|}{\left|x_{0}-y\right|^{n-\beta+1}} d y \\
& \leq C R \sum_{j=k+2}^{\infty} \int_{A_{j}} \frac{|f(y)|}{\left|x_{0}-y\right|^{n-\beta+1}} d y \\
& \leq C R \sum_{j=k+2}^{\infty} 2^{-j(n-\beta+1)} \int_{A_{j}}|f(y)| d y .
\end{aligned}
$$

Lemma 3.1 implies

$$
\int_{A_{j}}|f(y)| d y \leq C\left\|f \chi_{j}\right\|_{p(\cdot)}\left\|\chi_{B_{j}}\right\|_{p^{\prime}(\cdot)}
$$

Applying Lemma 3.3 we obtain

$$
\frac{\left(\left|x_{0}\right|+R\right)^{\alpha}}{|Q|^{\frac{\beta}{n}}\left\|\chi_{Q}\right\|_{p^{\prime}(\cdot)}} \int_{Q}\left|\tilde{I}_{\beta} f_{3}(x)-c_{3}\right| d x \leq C \frac{2^{k \alpha}}{|Q|^{\frac{\beta-1}{n}}} 2^{-k(n-\beta+1)} \sum_{j=-\infty}^{k-3}\left\|f \chi_{j}\right\|_{p(\cdot)}\left\|\chi_{B_{j}}\right\|_{p^{\prime}(\cdot)}\left\|\chi_{Q}\right\|_{p(\cdot) .} .
$$

Since $\alpha-\beta+\frac{n}{p^{+}}+1>0$, by (4.4) and (4.9),

$$
\begin{aligned}
\frac{\left(\left|x_{0}\right|+R\right)^{\alpha}}{|Q|^{\frac{\beta}{n}}\left\|\chi_{Q}\right\|_{p^{\prime}(\cdot)}} \int_{Q}\left|\tilde{I}_{\beta} f_{3}(x)-c_{3}\right| d x & \leq C \sum_{j=k+2}^{\infty} 2^{(k-j)\left(\alpha-\beta+\frac{n}{p^{+}}+1\right)} 2^{j \alpha}\left\|f \chi_{j}\right\|_{p(\cdot)} \\
& \leq C\|f\|_{K_{p(\cdot), q}^{\alpha}\left(\mathbb{R}^{n}\right)} .
\end{aligned}
$$

Combining (4.6)-(4.8), (4.10), and (4.11), case (3) is proved and then the proof of the theorem is completed.

Proof of Theorem 2.2 Let $f_{i}(x)=2^{-i \alpha} \chi_{\left[2^{i}, 2^{i}+1\right]}(x)$ for $i \geq 1$, then $\left\|f_{i}\right\|_{\dot{K}_{p(\cdot), q}^{\alpha}\left(\mathbb{R}^{1}\right)} \sim 1$ and

$$
\begin{aligned}
\tilde{I}_{\beta} f_{i}(x)-\tilde{I}_{\beta} f_{i}\left(2^{i}\right) & =\int_{\mathbb{R}^{1}}\left\{\frac{1}{|x-y|^{1-\beta}}-\frac{1}{\left|2^{i}-y\right|^{1-\beta}}\right\} f_{i}(y) d y \\
& =\frac{2^{-i \alpha}}{\beta}\left\{\left(2^{i}+1-x\right)^{\beta}-\left(2^{i}-x\right)^{\beta}-1\right\} .
\end{aligned}
$$

Let $B_{i}=\left(2^{i}-1,2^{i}\right)$ with $\left|B_{i}\right|=1$, then

$$
\frac{1}{\left|B_{i}\right|} \int_{B_{i}} \tilde{I}_{\beta} f_{i}(x) d x=\frac{2^{-i \alpha}\left(2^{\beta+1}-\beta-3\right)}{\beta(\beta+1)}+\tilde{I}_{\beta} f_{i}\left(2^{i}\right) .
$$

Hence

$$
\begin{aligned}
& \frac{1}{\left|B_{i}\right|} \int_{B_{i}}\left|\tilde{I}_{\beta} f_{i}(x)-\left(\tilde{I}_{\beta} f_{i}\right)_{B_{i}}\right| d x \\
& \quad=\frac{2^{-i \alpha}}{\beta} \int_{2^{i}-1}^{2^{i}}\left|\left(2^{i}+1-x\right)^{\beta}-\left(2^{i}-x\right)^{\beta}-1-\frac{2^{\beta+1}-\beta-3}{\beta+1}\right| d x
\end{aligned}
$$




$$
\begin{aligned}
& =\frac{2^{-i \alpha}}{\beta} \int_{0}^{1}\left|(2-x)^{\beta}-(1-x)^{\beta}-1-\frac{2^{\beta+1}-\beta-3}{\beta+1}\right| d x \\
& =C 2^{-i \alpha}
\end{aligned}
$$

and

$$
\begin{aligned}
& \lim _{i \rightarrow \infty} \frac{1}{\left(\left|2^{i}-\frac{1}{2}\right|+\frac{1}{2}\right)^{-\alpha-\varepsilon}\left|B_{i}\right|^{\beta+\varepsilon}\left\|\chi_{B_{i}}\right\|_{p^{\prime}(\cdot)}} \int_{B_{i}}\left|\tilde{I}_{\beta} f_{i}(x)-\left(\tilde{I}_{\beta} f_{i}\right)_{B_{i}}\right| d x \\
& \quad=\lim _{i \rightarrow \infty} \frac{C 2^{-i \alpha}}{\left(\left|2^{i}-\frac{1}{2}\right|+\frac{1}{2}\right)^{-\alpha-\varepsilon}\left|B_{i}\right|^{\beta+\varepsilon-1}\left\|\chi_{B_{i}}\right\|_{p^{\prime}(\cdot)}} \\
& \quad=\lim _{i \rightarrow \infty} C 2^{i \varepsilon}=\infty .
\end{aligned}
$$

This finishes the proof of Theorem 2.2.

\section{Competing interests}

The authors declare that they have no competing interests.

\section{Authors' contributions}

The authors worked jointly in drafting and approved the final manuscript.

\section{Acknowledgements}

The first author was supported by National Natual Science Foundation of China (11471033), Anhui Provincial Natural Science Foundation (1408085MA01) and University NSR Project of Anhui Province (KJ2014A087).

Received: 15 June 2015 Accepted: 21 December 2015 Published online: 04 January 2016

\section{References}

1. Gatto, A, Vagi, S: Integrals on spaces of homogeneous type. In: Analysis and Partial Differential Equations. Lect. Notes in Pure and Appl. Math., vol. 122, pp. 171-216. Dekker, New York (1990)

2. Harboure, E, Salinas, O, Viviani, B: Boundedness of the fractional integral on weighted Lebesgue and Lipschitz spaces. Trans. Am. Math. Soc. 349(1), 235-255 (1997)

3. Ramseyer, M, Salinas, O, Viviani, B: Lipschitz type smoothness of the fractional integral on variable exponent spaces. J. Math. Anal. Appl. 403(1), 95-106 (2013)

4. Cruz-Uribe, D, Fiorenza, A: Variable Lebesgue Spaces: Foundations and Harmonic Analysis. Springer, Heidelberg (2013)

5. Diening, L, Harjulehto, P, Hästö, P Růžička, M: Lebesgue and Sobolev Spaces with Variable Exponents. LNM Series, vol. 2017. Springer, Berlin (2011)

6. Peetre, J: On the theory of $\mathcal{L}_{p, \lambda}$ spaces. Funct. Anal. Appl. 4, 71-87 (1969)

7. Almeida, A, Drihem, D: Maximal, potential and singular type operators on Herz spaces with variable exponents. J. Math. Anal. Appl. 394, 781-795 (2012)

8. Samko, S: Variable exponent Herz spaces. Mediterr. J. Math. 10, 2007-2025 (2013)

9. Izuki, M: Herz and amalgam spaces with variable exponent, the Haar wavelets and greediness of the wavelet system. East J. Approx. 15, 87-109 (2009)

10. Lu, SZ, Yang, DC, Hu, GE: Herz Type Spaces and Their Applications. Science Press, Beijing (2008)

11. Kováčik, O, Rákosník, J: On spaces $L^{p(x)}$ and $W^{k, p(x)}$. Czechoslov. Math. J. 41, 592-618 (1991)

12. Nekvinda, A: Hardy-Littlewood maximal operator on $L^{p(x)}\left(\mathbb{R}^{n}\right)$. Math. Inequal. Appl. 7, 255-265 (2004)

13. Cruz-Uribe, D, Fiorenza, A, Martell, JM: The boundedness of classical operators on variable $L^{p}$ spaces. Ann. Acad. Sci. Fenn., Math. 31(1), 239-264 (2006) 